\title{
RIESZ DECOMPOSITION PROPERTY IMPLIES ASYMPTOTIC PERIODICITY OF POSITIVE AND CONSTRICTIVE OPERATORS
}

\author{
WOJCIECH BARTOSZEK
}

(Communicated by Palle E. T. Jorgensen)

\begin{abstract}
Consider a linear and positive operator $\mathbf{T}$ acting on an ordered, $F$-normed linear space $\mathbf{X}$. Assume that there exists an open neighborhood $\mathbf{U} \ni \mathbf{0}$ such that the trajectory $\left\{\mathbf{T}^{n}(\mathbf{x})\right\}$ is attracted to a compact set $\mathbf{F}_{\mathbf{U}}$ whenever $\mathbf{x}$ is taken from $\mathbf{U}$ and that the positive cone $\mathbf{X}_{+}$is closed, proper, and reproducing. It is shown that if $\left(\mathbf{X}, \mathbf{X}_{+}\right)$has the Riesz Decomposition Property then $\mathbf{T}$ has asymptotically periodic iterates.
\end{abstract}

\section{INTRODUCTION}

Asymptotic periodicity of iterates of positive contractions acting on Banach lattices was investigated by several authors in the last decade (see [LLY, V, B, $\mathrm{K}]$ ). It was proved in [B] that if a linear positive contraction $\mathbf{T}$ acting on a real Banach lattice $\mathbf{X}$ is constrictive (see the definition below) then there exist a sequence of positive normalized vectors $\mathbf{y}_{1}, \ldots, \mathbf{y}_{r}$ in $\mathbf{X}$ and a sequence of positive (bounded) linear functionals $\Lambda_{1}, \ldots, \Lambda_{r}$ on $\mathbf{X}$ such that for each $\mathbf{x} \in \mathbf{X}$ one has

$$
\mathbf{T}^{n}(\mathbf{x})=\sum_{j=1}^{r} \Lambda_{j}(\mathbf{x}) \mathbf{y}_{\alpha^{n}(j)}+\mathbf{R}_{n}(\mathbf{x})
$$

where $\alpha$ is some permutation of the set $\{1, \ldots, r\}$ and the remainder $\mathbf{R}_{n}(\mathbf{x}) \rightarrow$ $\mathbf{0}$ in the norm. An operator $\mathbf{T}$ satisfying (1) is called asymptotically periodic.

The first attempt to extend this result to constrictive operators acting on ordered Banach spaces was made by Miklavčič in [M]. He has considered a power bounded operator $\mathbf{T}$, acting on a Banach space $\mathbf{X}$, that preserves some fixed cone $\mathbf{X}_{+} \subset \mathbf{X}$ (i.e., $\mathbf{T}\left(\mathbf{X}_{+}\right) \subseteq \mathbf{X}_{+}$). He has imposed on the cone $\mathbf{X}_{+}$ some extra conditions that allow him to establish the decomposition (1). However, we would like to notice that the ordered Banach spaces considered in [M] are actually Banach lattices. In fact, the condition (A2) in [M] implies that $|\mathbf{x}|=\mathbf{x}_{+}+\mathbf{x}_{-}$is the modulus in $\left(\mathbf{X}, \mathbf{X}_{+}\right)$. Moreover the new norm

Received by the editors February 9, 1991.

1991 Mathematics Subject Classification. Primary 46A06, 46B30, 47B50.

Key words and phrases. Asymptotic periodicity, positive operator, Riesz Decomposition Property. 
$\|\mathbf{x}\|_{1}=\sup \left\{\left\|\mathbf{T}^{n}(|\mathbf{x}|)\right\|: n \geq 0\right\}$ is equivalent to the old one, $\mathbf{T}$ is a contraction with respect to it, and $\left(\mathbf{X}, \mathbf{X}_{+},\|\cdot\|_{1}\right)$ is a Banach lattice.

The aim of this paper is to find some essentially weaker conditions than Miklavčič's assumption (A2) in [M] that still guarantee the asymptotic periodicity of trajectories. For instance it is easy to see that every positive and constrictive linear operator acting on the ordered Banach space $\mathbf{C}^{1}([0,1])$ (with the positive cone $\mathbf{C}_{+}^{1}([0,1])=\left\{f \in \mathbf{C}^{1}([0,1]): f(x) \geq 0\right.$ for every $\left.\left.x \in[0,1]\right\}\right)$ is asymptotically periodic. To show this, let us first remark that in this case every constrictive and positive linear operator $\mathbf{T}$ can be extended easily to a positive and constrictive operator $\widetilde{\mathbf{T}}$ acting on the Banach lattice $\mathbf{C}([0,1])$. By the previous remarks we may assume that $\widetilde{\mathbf{T}}$ is a contraction, so Theorem 1 in [B] (or Theorem 2.3 in [M]) implies that all trajectories $\widetilde{\mathbf{T}}^{n} f$ (where $f$ is from $\mathbf{C}([0,1]))$ have finite $\omega$-limit sets. An easy density argument shows that all these $\omega$-limit sets are included in the Banach space $\mathbf{C}^{\mathbf{l}}([0,1])$. In particular we obtain the asymptotic periodicity of $\mathbf{T}$ in $\mathbf{C}^{\mathbf{l}}([0,1])$.

Motivated by the above we investigate asymptotic periodicity of constrictive and positive operators on ordered $F$-spaces with Riesz Decomposition Property (abbreviated by R.D.P.). It is well known that R.D.P. is essentially weaker than lattice axioms. We find this property to be crucial property of the pair $\left(\mathbf{X}, \mathbf{X}_{+}\right)$ that makes the asymptotic periodicity possible for positive and constrictive operators.

At the end of the note, we deal with positive and constrictive linear operators on the Banach lattice $\mathbf{C}^{2}([0,1])$. In particular, some properties of positive and finite-dimensional projections on this space are found.

2.

Let $\mathbf{X}$ be a linear space over real numbers. A subset $\mathbf{X}_{+} \subseteq \mathbf{X}$ is said to be a (proper) cone (with vertex $\mathbf{0}$ ) if it satisfies the following conditions: if $\mathbf{x} \in \mathbf{X}_{+}$ and $-\mathbf{x} \in \mathbf{X}_{+}$then $\mathbf{x}=\mathbf{0}$; and if $\mathbf{x} \in \mathbf{X}_{+}$and $t \geq 0$ then $t \mathbf{x} \in \mathbf{X}_{+}$; and if $\mathbf{x}, \mathbf{y} \in \mathbf{X}_{+}$then $\mathbf{x}+\mathbf{y} \in \mathbf{X}_{+}$. Every cone $\mathbf{X}_{+}$induces a partial ordering on $\mathbf{X}$ by $\mathbf{x} \leq \mathbf{y}$ if $\mathbf{y}-\mathbf{x} \in \mathbf{X}_{+}$. A linear operator $\mathbf{T}: \mathbf{X} \rightarrow \mathbf{X}$ is called positive if $\mathbf{T}(\mathbf{x}) \leq \mathbf{T}(\mathbf{y})$ whenever $\mathbf{x} \leq \mathbf{y}$. We always assume that our cone is generating (i.e., $\mathbf{X}=\mathbf{X}_{+}-\mathbf{X}_{+}$). Let, in the space $\mathbf{X}$, be given an $F$-norm $\|\cdot\|$ that makes the space $\mathbf{X}$ a complete $\mathbf{F}$-space (see [R] for the definition). All operators considered in this note are linear and continuous with respect to the fixed $F$ norm $\|\cdot\|$ on $\mathbf{X}$. We also assume that the cone $\mathbf{X}_{+}$is an $F$-norm closed subset of $\mathbf{X}$. By $\mathbf{X}^{*}$ we denote the vector space of all continuous, real functionals on $\mathbf{X}$ and By $\mathbf{T}^{*}$ the adjoint operator to $\mathbf{T}$. The dual cone $\mathbf{X}_{+}^{*}$ is defined to be the set of all functionals $\Lambda \in \mathbf{X}^{*}$ such that $\Lambda(\mathbf{x}) \geq \mathbf{0}$ for all $\mathbf{x} \in \mathbf{X}_{+}$. We say that an ordered vector space $\left(\mathbf{X}, \mathbf{X}_{+}\right)$has the Riesz Decomposition Property (R.D.P.) if for every $\mathbf{u}, \mathbf{v}, \mathbf{w} \in \mathbf{X}_{+}$with $\mathbf{u} \leq \mathbf{v}+\mathbf{w}$ there exist $\mathbf{u}_{\mathbf{v}}, \mathbf{u}_{\mathbf{w}} \in \mathbf{X}_{+}$ such that $\mathbf{u}_{\mathbf{v}} \leq \mathbf{v}$, and $\mathbf{u}_{\mathbf{w}} \leq \mathbf{w}$, and $\mathbf{u}=\mathbf{u}_{\mathbf{v}}+\mathbf{u}_{\mathbf{w}}$. It is well known that all Riesz spaces (in particular all Banach lattices) have the R.D.P. The set of all order bounded functionals on $\mathbf{X}$ is denoted by $\mathbf{X}^{b}$ (see [KN] for definitions and more details). The classical Riesz Theorem asserts that if $\mathbf{X}$ has the R.D.P. then $\mathbf{X}^{b}$ is a Riesz vector space. In particular, if $\Omega$ is a finite-dimensional subspace of $\mathbf{X}$, ordered by the generating cone $\Omega_{+}=\mathbf{X}_{+} \cap \Omega$, and $\left(\Omega, \Omega_{+}\right)$has the R.D.P., then $\left(\Omega, \Omega_{+}\right)$is actually a vector lattice. In fact, by the above-mentioned Riesz 
Theorem, $\left(\Omega^{*}, \Omega_{+}^{*}\right)$ is a vector lattice. Applying it once again to the dual space we have that $\left(\Omega^{* *}, \Omega_{+}^{* *}\right)$ is a vector lattice, as is $\left(\Omega, \Omega_{+}\right)$. Finally it should be added that in a finite-dimensional case each proper closed generating cone is normal, so the order dual coincides with the algebraic dual and the Riesz Theorem is applicable here.

Definition 2.1. A linear operator $\mathbf{T}: \mathbf{X} \rightarrow \mathbf{X}$ is called constrictive if there exist an open ball $\mathbf{K}(\mathbf{0}, r)=\{\mathbf{x} \in \mathbf{X}:\|\mathbf{x}\|<r\}$ and a compact set $\mathbf{F} \subseteq \mathbf{X}$ such that for every $\mathbf{x} \in \mathbf{K}(\mathbf{0}, r)$ the trajectory $\mathbf{T}^{n}(\mathbf{x})$ is attracted to $\mathbf{F}$ (i.e., $\inf \left\{\left\|\mathbf{T}^{n}(\mathbf{x})-\mathbf{f}\right\|\right.$ : $\left.\mathbf{f} \in \mathbf{F}\} \rightarrow_{n \rightarrow \infty} 0\right)$.

Remark 2.1. For a Banach space, where the $F$-norm is simply a norm, it is enough to consider only $r=1$ in the above definition (compare [LLY, B] or [M]). Moreover, the homogeneity of the norm implies that $\mathbf{T}$ is constrictive if and only if for every bounded set $\mathbf{B} \subseteq \mathbf{X}$ there exists a compact set $\mathbf{F}_{\mathbf{B}}$ such that every trajectory $\mathbf{T}^{n}(\mathbf{x})$, which starts from a point $\mathbf{x} \in \mathbf{B}$, is attracted to $\mathbf{F}_{\mathbf{B}}$. For general $F$-spaces the definition of constrictivity should go through open balls rather than bounded sets. For instance, in the $F$-space $\mathbf{X} \in \mathbb{R}^{\mathbb{N}}$ equipped with the $F$-norm

$$
\left\|\left(x_{j}\right)\right\|=\sum_{j=1}^{\infty} \frac{1}{2^{j}} \frac{\left|x_{j}\right|}{1+\left|x_{j}\right|}
$$

every bounded and closed set is automatically compact. So, if constrictivity of operators were defined by bounded sets, the identity operator would be constrictive here. Our intention is to give such a definition of constrictivity that iterates of a constrictive operator are asymptotically close to compact (finitedimensional) operators. In sequel we will find Definition 2.1 to be an appropriate generalization of constrictivity in general $F$-spaces.

Now let $\mathbf{T}$ be a constrictive operator on $\mathbf{X}$. Since the completness of the $F$-norm is assumed, thus by the Mazur-Orlicz Theorem (see also [R, p. 39]) the family of iterates $\left\{\mathbf{T}^{n}: n \geq 0\right\}$ is equicontinuous, i.e.,

$$
\forall_{\varepsilon>0} \exists_{\delta>0} \sup \left\{\left\|\mathbf{T}^{n}(\mathbf{x})\right\|:\|\mathbf{x}\| \leq \delta, n \geq 0\right\} \leq \varepsilon .
$$

The $\omega$-limit set of the vector $\mathbf{x}$ from $\mathbf{X}$ is defined to be the set $\omega(\mathbf{x})=\{\mathbf{y} \epsilon$ $\mathbf{X}: \mathbf{y}=\lim \mathbf{T}^{n_{k}}(\mathbf{x})$ for some sequence $\left.n_{k} \nearrow \infty\right\}$. Observe that if $\mathbf{T}$ is constrictive, then every trajectory $\gamma(\mathbf{x})=\left\{\mathbf{T}^{n}(\mathbf{x}): n \geq 0\right\}$ is a relatively compact subset of $\mathbf{X}$, so for each $\mathbf{x}$ the $\omega$-limit set $\omega(\mathbf{x})$ is nonempty, compact, and T-invariant (i.e., $\mathbf{T}(\omega(\mathbf{x})) \subseteq \omega(\mathbf{x}))$. The following lemma, which gathers some properties of the dynamical system $(\omega(\mathbf{x}), \mathbf{T})$, is essentially due to Dafermos and Slemrod (see [DS, p. 98]).

Lemma 2.1. If $\mathbf{T}$ is a constrictive operator on $\mathbf{X}$ then

(2.1) $\mathbf{y} \in \omega(\mathbf{x}) \Rightarrow \omega(\mathbf{y})=\omega(\mathbf{x})$;

(2.2) $\left.\mathbf{T}\right|_{\omega(\mathbf{x})}$ is an invertible mapping such that the family $\left\{\left.\mathbf{T}\right|_{\omega(\mathbf{x})} ^{n}: n \in \mathbb{Z}\right\}$ is equicontinuous on $\omega(\mathbf{x})$.

Remark 2.2. Dafermos and Slemrod have proved (2.1) and (2.2) for arbitrary nonexpansive (not necessarily constrictive or linear) mappings acting on a complete metric space. Since they considered nonexpansive mappings, (2.2) had the following nicer version: $\left.\mathbf{T}\right|_{\omega(\mathbf{x})}$ is an invertible isometry. If just equicontinuity of $\mathbf{T}^{n}$ is given we cannot expect an isometry effect on $\omega(\mathbf{x})$. However if the 
space $\mathbf{X}$ was appropriately renormed then it would be possible to have it. The proof of Lemma 2.1 is standard and may be modeled on the original proof of Theorem 1 in [DS].

Now let us set $\Omega=\bigcup_{\mathbf{x} \in \mathbf{X}} \omega(\mathbf{x})$. It is easy to notice that $\Omega$ is a closed and T-invariant subset of $\mathbf{X}$. Moreover, because of Lemma 2.1, the properties of the dynamical system $(\Omega, T)$ are also very nice. We collect them in the next lemma. Some of these properties were presented in [B], but only in the case when $\mathbf{T}$ was a positive and constrictive contraction on a Banach lattice. For the reader's convenience a complete proof is presented here.

Lemma 2.2. Let $\mathbf{T}$ be a constrictive operator on an $F$-space $\mathbf{X}$. Then

(2.3) $\Omega$ is a finite-dimensional subspace of $\mathbf{X}$;

(2.4) $\left.\mathrm{T}\right|_{\Omega}$ has the inverse on $\Omega$;

(2.5) the family $\left\{\left.\mathbf{T}\right|_{\Omega} ^{n}: n \in \mathbb{Z}\right\}$ is equicontinuous on $\Omega$;

(2.6) if $\mathbf{T}\left(\mathbf{X}_{+}\right) \subseteq \mathbf{X}_{+}$for some proper closed generating cone $\mathbf{X}_{+} \subseteq \mathbf{X}$ then $\left.\mathbf{T}\right|_{\Omega} ^{n}\left(\Omega_{+}\right) \subseteq \Omega_{+}$for arbitrary integer $n$ where $\Omega_{+}=\mathbf{X}_{+} \cap \Omega$ is a closed, proper, and generating cone in $\Omega$;

(2.7) if $\mathbf{T}\left(\mathbf{X}_{+}\right) \subseteq \mathbf{X}_{+}$and $\left(\mathbf{X}, \mathbf{X}_{+}\right)$has the R.D.P. then $\left(\Omega, \Omega_{+}\right)$has the R.D.P. as well.

Proof. (2.3) For every $\mathbf{x}, \mathbf{y} \in \Omega$ there exists a sequence $n_{k} \nearrow \infty$ such that $\mathbf{T}^{-n_{k}}(\mathbf{x}), \mathbf{T}^{-n_{k}}(\mathbf{y})$ converge in $\Omega$ to $\mathbf{x}^{\prime}, \mathbf{y}^{\prime}$ respectively. By (2.2) and properties of the $F$-norm we have

$$
\left\|\mathbf{T}^{n_{k}}\left(\mathbf{x}^{\prime}+\mathbf{y}^{\prime}\right)-(\mathbf{x}+\mathbf{y})\right\| \leq\left\|\mathbf{T}^{n_{k}}\left(\mathbf{x}^{\prime}-\mathbf{T}^{-n_{k}}(\mathbf{x})\right)\right\|+\left\|\mathbf{T}^{n_{k}}\left(\mathbf{y}^{\prime}-\mathbf{T}^{-n_{k}}(\mathbf{y})\right)\right\| \rightarrow 0 .
$$

This proves $\mathbf{x}+\mathbf{y} \in \Omega$. Clearly if $\mathbf{x} \in \Omega$ then $t \mathbf{x} \in \Omega$ for every scalar $t$. It follows that $\Omega$ is a linear subspace of $\mathbf{X}$. Now let $U=\Omega \cap \mathbf{K}(\mathbf{0}, r)$ be an open ball in $\Omega$. Since for every $\mathbf{x} \in \mathbf{U}$ we have simutaneously $\mathbf{T}^{n_{k}}(\mathbf{x}) \rightarrow \mathbf{x}$ for some $n_{k} \nearrow \infty$ (compare (2.1)) and $\mathbf{T}^{n}(\mathbf{x}) \rightarrow \mathbf{F}$ compact $\mathbf{U} \subseteq \mathbf{F}$. In particular, $\mathbf{U}$ must be a totally bounded neighborhood of 0 in $\Omega$, so $\Omega$ is finite dimensional (see [KN, p. 62]).

(2.4) It has been noticed that $\mathbf{T}$ has the inverse on every $\omega$-limit set $\omega(\mathbf{x})$. Hence $\left.\mathbf{T}\right|_{\Omega}$ is invertible as it is a linear operator "onto" acting on a finitedimensional vector space $\Omega$.

(2.5) We have to prove that for every $\varepsilon>0$ there exists $\delta>0$ such that $\left\|\left.\mathbf{T}\right|_{\Omega} ^{n}(\mathbf{x})\right\| \leq \varepsilon$ if $\|\mathbf{x}\| \leq \delta$ and $n \in \mathbb{Z}$. It is easy (because of the mentioned Mazur and Orlicz Theorem) that if only positive $n$ is considered then for every $\varepsilon>0$ there exists a suitable $\delta>0$. Now let $n \in \mathbb{Z}$ be arbitrary and $\|\mathbf{x}\| \leq \delta$. Since $\mathbf{T}^{n_{k}}(\mathbf{x}) \rightarrow \mathbf{x}$ for some $n_{k} \nearrow \infty$,

$$
\left.\mathbf{T}\right|_{\Omega} ^{n}(\mathbf{x})=\left.\lim _{k \rightarrow \infty} \mathbf{T}\right|_{\Omega} ^{n}\left(\left.\mathbf{T}\right|_{\Omega} ^{n_{k}}(\mathbf{x})\right)=\left.\lim _{k \rightarrow \infty} \mathbf{T}\right|_{\Omega} ^{n_{k}+n}(\mathbf{x})
$$

so $\left\|\left.\mathbf{T}\right|_{\Omega} ^{n}(\mathbf{x})\right\| \leq \varepsilon$ since $n_{k}+n$ is positive when $k$ is large enough.

(2.6) By the closedness of the cone $\mathbf{X}_{+}$the inclusion $\left.\mathbf{T}\right|_{\Omega} ^{n}\left(\Omega_{+}\right) \subseteq \Omega_{+}$is obvious for positive $n$. The similar argument as in the previous point shows that it is valid for arbitrary integer $n$. Clearly $\Omega_{+}$is closed as an intersection of two closed sets, and proper as a subset of a proper cone $\mathbf{X}_{+}$. Since every $\mathbf{x} \in \Omega$ has a representation $\mathbf{x}=\lim _{k \rightarrow \infty} T^{n_{k}}\left(\mathbf{x}_{+}^{\prime}-\mathbf{x}_{-}^{\prime}\right)=\mathbf{x}_{+}-\mathbf{x}_{-}$where $\mathbf{x}_{+}$and $\mathbf{x}_{-}$are in $\Omega_{+}$, the cone $\Omega_{+}$is generating for $\Omega$. 
(2.7) Assume that $\left(\mathbf{X}, \mathbf{X}_{+}\right)$has the R.D.P. and $\mathbf{x}_{0} \leq \mathbf{x}_{1}+\mathbf{x}_{2}$ for some $\mathbf{x}_{0}, \mathbf{x}_{1}$, $\mathbf{x}_{2} \in \Omega_{+}$. Since all orbits $\left\{\left.\mathbf{T}\right|_{\Omega} ^{n}\left(\mathbf{x}_{j}\right): n \in \mathbb{Z}\right\} \quad(j=0,1,2)$ are precompact, we may choose $n_{k} \nearrow \infty$ such that $\left.\lim _{k \rightarrow \infty} \mathbf{T}\right|_{\Omega} ^{-n_{k}}\left(\mathbf{x}_{j}\right)=\mathbf{y}_{j} \in \Omega_{+}$for $j=0,1,2$. Clearly $\mathbf{y}_{0} \leq \mathbf{y}_{1}+\mathbf{y}_{2}$ by the positivity of $\mathbf{T}$ and closedness of $\Omega_{+}$. Since $\left(\mathbf{X}, \mathbf{X}_{+}\right)$has the R.D.P., there are $\mathbf{z}_{1}, \mathbf{z}_{2} \in \mathbf{X}_{+}$such that $\mathbf{z}_{j} \leq \mathbf{y}_{j}(j=1,2)$ and $\mathbf{y}_{0}=\mathbf{z}_{1}+\mathbf{z}_{2}$. Choosing a subsequence of $n_{k}$ if necessary we may guarantee the convergence of sequences $\left.\mathbf{T}\right|_{\Omega} ^{n_{k}}\left(\mathbf{z}_{j}\right)(j=1,2)$. Set $\mathbf{x}_{j}^{\prime}=\left.\lim _{k \rightarrow \infty} \mathbf{T}\right|_{\Omega} ^{n_{k}}\left(\mathbf{z}_{j}\right) \in \Omega_{+}$ and let us notice that equicontinuity of the family $\left\{\left.\mathbf{T}\right|_{\Omega} ^{n}: n \in \mathbb{Z}\right\}$ implies that $\mathbf{x}_{j}^{\prime} \leq \mathbf{x}_{j}$ and $\mathbf{x}_{0}=\mathbf{x}_{1}^{\prime}+\mathbf{x}_{2}^{\prime}$ as well.

Following immediately is

Corollary 2.1. Let $\mathbf{T}$ be a constrictive operator on an ordered $F$-space $\mathbf{X}$ with a closed, proper, and generating cone $\mathbf{X}_{+}$. If $\mathbf{T}$ is positive and the space $\mathbf{X}$ has the R.D.P. then the set of all T-recurrent vectors $\Omega$ is finite-dimensional Riesz space for the ordering inherited from $\mathbf{X}$. Moreover $\left.\mathbf{T}\right|_{\Omega}$ has a positive inverse $\left.\mathbf{T}\right|_{\Omega} ^{-1}$ on $\Omega$.

Convention. In the sequel, if $\mathbf{x} \in \Omega$ and $n \in \mathbb{Z}$ then $\left.\mathbf{T}\right|_{\Omega} ^{n}(\mathbf{x})$ is abbreviated simply to $\mathbf{T}^{n}(\mathbf{x})$.

To formulate the next results let us recall the following:

Definition 2.2. A nonzero positive element $y$ of an ordered vector space $\left(\mathbf{Y}, \mathbf{Y}_{+}\right)$is called an atom if the inequality $\mathbf{0} \leq \mathbf{z} \leq \mathbf{y}$ holds for only $\mathbf{z}=t \mathbf{y}$ where $t \in[0,1]$.

Definition 2.3. Two positive vectors $y_{1}$ and $y_{2}$ from an ordered vector space $\left(\mathbf{Y}, \mathbf{Y}_{+}\right)$are said to be orthogonal if $\mathbf{0} \leq \mathbf{z} \leq \mathbf{y}_{j}(j=1,2)$ imply $\mathbf{z}=\mathbf{0}$.

Lemma 2.3. Let $\mathbf{T}$ be a positive and constrictive linear operator on an ordered $F$ space $\left(\mathbf{X}, \mathbf{X}_{+}\right)$. If $\mathbf{X}$ has the R.D.P. then there exists a linear base $\left\{\mathbf{y}_{1}, \ldots, \mathbf{y}_{r}\right\}$ in $\Omega(r=\operatorname{dim}(\Omega))$ with the following properties:

(2.8) each vector $\mathbf{y}_{j}$ is an atom of $\left(\Omega, \Omega_{+}\right)$;

(2.9) base vectors $\mathbf{y}_{j}$ are pairwise orthogonal in $\left(\Omega, \Omega_{+}\right)$;

(2.10) $\mathbf{x} \in \Omega_{+}$if and only if $\mathbf{x}=\sum_{j=1}^{r} t_{j} \mathbf{y}_{j}$ for some nonnegative $t_{j}$;

(2.11) there are strictly positive scalars $\gamma_{j}(j=1, \ldots, r)$ and a permutation $\alpha$ of the set $\{1, \ldots, r\}$ such that $\mathbf{T}\left(\mathbf{y}_{j}\right)=\gamma_{j} \mathbf{y}_{\alpha(j)} ;$

(2.12) there exists a natural number $d$ such that $\left.\mathbf{T}\right|_{\Omega} ^{d}=\left.\mathbf{I d}\right|_{\Omega}$.

Proof. (2.8), (2.9), and (2.10) follow from Theorem 26.11 in [LZ].

(2.11) If $\mathbf{T}\left(\mathbf{y}_{j}\right)$ were not an atom in $\left(\Omega, \Omega_{+}\right)$then $\mathbf{T}\left(\mathbf{y}_{j}\right)=\sum_{k=1}^{r} t_{k} \mathbf{y}_{k}$ for at least two positive $t_{k}$ and $t_{l}$. In particular $\mathbf{T}\left(\mathbf{y}_{j}\right) \geq t_{i} \mathbf{y}_{i}$ for $i=k, l$. Since the operator $\left.\mathbf{T}\right|_{\Omega} ^{-1}$ is positive, $\mathbf{y}_{j} \geq t_{i} \mathbf{T}^{-1}\left(\mathbf{y}_{i}\right) \quad(i=k, l)$. The atomness of $\mathbf{y}_{j}$ implies $\mathbf{T}^{-1}\left(\mathbf{y}_{i}\right)=\tilde{t}_{i} \mathbf{y}_{j} \quad(i=k, l)$, which contradicts $\left.\mathbf{T}\right|_{\Omega}$ is an isomorphism of $\Omega$. Hence in the expansion $\mathbf{T}\left(\mathbf{y}_{j}\right)=\sum_{k=1}^{r} t_{k} \mathbf{y}_{k}$ only one term (let us denote it by $\left.t_{\alpha(j)} \mathbf{y}_{\alpha(j)}=\gamma_{j} \mathbf{y}_{\alpha(j)}\right)$ is nonzero and (2.11) is proved.

(2.12) Now let $d$ be such a natural number that $\alpha^{d}=\mathrm{id}$ where $\alpha$ is the permutation described in (2.11). Iterating (2.11) we get $\mathbf{T}^{d}\left(\mathbf{y}_{j}\right)=\beta_{j} \mathbf{y}_{j}$ where $\beta_{j}=\gamma_{j} \gamma_{\alpha(j)} \cdots \gamma_{\alpha^{d-1}(j)}$. To finish the proof it is enough to show that $\beta_{j}=1$ 
for every $j=1, \ldots, r$. But if $\beta_{j} \neq 1$ then the orbit $\left\{\mathbf{T}^{n d}\left(\mathbf{y}_{j}\right): n \in \mathbb{Z}\right\}$ is not relatively compact, which contradicts the constrictivity assumption.

Remark 2.3. Let $\left\{j, \alpha(j), \ldots, \alpha^{p}(j)\right\}$ be a cycle of the permutation $\alpha$ (i.e., $\alpha^{p+1}(j)=j$ and $p$ is the smallest with this property). It is clear that if the base vectors $\mathbf{y}_{\alpha^{\prime}(j)}, l=0,1, \ldots, p$, are replaced by $\tilde{\mathbf{y}}_{\alpha^{\prime}(j)}=\mathbf{T}^{l}\left(\mathbf{y}_{j}\right)$ where $l=0, \ldots, p$ then the coefficients $\tilde{\gamma}_{\alpha^{\prime}(j)}=1$. Since the permutation $\alpha$ can be decomposed on disjoint cycles, we are able to manage such a base $\mathbf{y}_{1}, \ldots, \mathbf{y}_{r}$ in $\Omega$ that all coefficients $\gamma_{j}$ in (2.11) are 1, and we assume that in the sequel our bases in $\Omega$ have this property.

Remark 2.4. The modulus of $\mathbf{x} \in \Omega$ is denoted by $|\mathbf{x}|_{\Omega}$. If $\mathbf{X}$ is a Banach lattice (or at least a Riesz space) then $|\mathbf{x}|_{\Omega}$ can be represented as $\lim _{n \rightarrow \infty} \mathbf{T}^{n d}(|\mathbf{x}|)$ where $d$ is the natural number from (2.12) and $|\cdot|$ denotes the modulus in $\mathbf{X}$ (compare [B, Proposition 1]). In general when $\left(\mathbf{X}, \mathbf{X}_{+}\right)$has only the R.D.P. the modulus $|\cdot|$ may not be defined on the whole $\mathbf{X}$. But even in this case $|\cdot|_{\Omega}$ is well described by the order $\leq$ and asymptotic behaviour of $\mathbf{T}^{n}$. To show this let us consider $F$-closed sets $\Gamma_{\mathbf{x}}=\left\{\lim _{n \rightarrow \infty} T^{n d}(\mathbf{y}): \mathbf{x} \leq \mathbf{y}\right.$ and $\left.-\mathbf{x} \leq \mathbf{y}\right\} \subseteq \Omega_{+}$ for $x \in \Omega$. By the positivity of $\mathbf{T}$ every set $\Gamma_{\mathbf{x}}$ is closed under minimum $\wedge_{\Omega}$. It follows that the infimum (with respect to $\leq$ ) of $\Gamma_{\mathbf{x}}$ is well defined and belongs to $\Gamma_{\mathbf{x}}$, so $|\mathbf{x}|_{\Omega} \leq \inf \left(\Gamma_{\mathbf{x}}\right)$. Obviously $|\mathbf{x}|_{\Omega} \in \Gamma_{\mathbf{x}}$, so the opposite inequality holds as well. Finally we get $|\mathbf{x}|_{\Omega}=\inf \left(\Gamma_{\mathbf{x}}\right)$.

Now we are in position to formulate and prove the main result of this note. We base the proof on the method of Lasota, Li, and Yorke [LLY, Lemma 5.2]. For the reader's convenience a complete proof is presented here.

Theorem. Let $\mathbf{T}$ be a positive, constrictive, and linear operator on an ordered $F$-space $\mathbf{X}$. Assume that the positive cone $\mathbf{X}_{+}$is generating, closed, proper, and has the R.D.P. Then there exist a family of positive vectors $\mathbf{y}_{1}, \ldots, \mathbf{y}_{r}$ in $\mathbf{X}, a$ family of positive linear functionals $\Lambda_{1}, \ldots, \Lambda_{r}$ in $\mathbf{X}^{*}$, and a permutation $\alpha$ of the set $\{1, \ldots, r\}$ that

(i) $\mathbf{T}\left(\mathbf{y}_{j}\right)=\mathbf{y}_{\alpha(j)}$ for every index $j \in\{1, \ldots, r\}$;

(ii) for every $\mathbf{x} \in \mathbf{X}$ there is the decomposition

$$
\mathbf{T}^{n}(\mathbf{x})=\sum_{j=1}^{r} \Lambda_{j}(\mathbf{x}) \mathbf{y}_{\alpha^{n}(j)}+\mathbf{R}_{n}(\mathbf{x})
$$

where $\mathbf{R}_{n}(\mathbf{x}) \rightarrow \mathbf{0}$ in the $F$-norm $\|\cdot\|$;

(iii) $\Lambda_{i}\left(\mathbf{y}_{k}\right)=\delta_{i k}$ for each pair of indices $i, k$ ( $\delta_{i k}$ denotes the Kronecker delta);

(iv) $\mathbf{T}^{*}\left(\Lambda_{k}\right)=\Lambda_{\alpha^{-1}(k)}$ for every $k \in\{1, \ldots, r\}$;

(v) for every $\mathbf{x}^{*} \in \mathbf{X}^{*}$ there is the decomposition

$$
\mathbf{T}^{* n}\left(\mathbf{x}^{*}\right)=\sum_{j=1}^{r} \mathbf{x}^{*}\left(\mathbf{y}_{j}\right) \Lambda_{\alpha^{-n}(j)}+\mathbf{R}_{n}^{*}\left(\mathbf{x}^{*}\right)
$$

where $\mathbf{R}_{n}^{*}\left(\mathbf{x}^{*}\right) \rightarrow \mathbf{0}$ in the weak* topology on $X^{*}$.

Proof. By Lemma 2.3 and Remark 2.3 there is an atomic base $\left\{\mathbf{y}_{1}, \ldots, \mathbf{y}_{r}\right\}$ in $\Omega$ and a permutation $\alpha$ of the set $\{1, \ldots, r\}$ such that $\mathbf{T}\left(\mathbf{y}_{j}\right)=\mathbf{y}_{\alpha(j)}$, so (i) is obvious. 
(ii) By constrictivity of $\mathbf{T}$ for every $x \in \mathbf{X}$ there is a sequence $n_{k} \nearrow \infty$ and scalars $\xi_{j}(\mathbf{x})(j=1, \ldots, r)$ such that $\mathbf{T}^{n_{k}}(\mathbf{x}) \rightarrow \sum_{j=1}^{r} \xi_{j}(\mathbf{x}) \mathbf{y}_{j}$. Clearly, if $\mathbf{x} \in \mathbf{X}_{+}$then for every $j$ the coefficient $\xi_{j}(\mathbf{x})$ is nonnegative. We may assume that the sequence $n_{k}$ has the following form $n_{k}=s+d m_{k}$ (if no we choose a suitable subsequence) where $m_{k} \nearrow \infty$ and $0 \leq s \leq d-1$. Now for arbitrary $m \in \mathbb{N}$ we have

$$
\mathbf{T}^{s+d m}(\mathbf{x})-\sum_{j=1}^{r} \xi_{j}(\mathbf{x}) \mathbf{y}_{j}=\mathbf{T}^{d\left(m-m_{k}\right)}\left(\mathbf{T}^{s+d m_{k}}(\mathbf{x})-\sum_{j=1}^{r} \xi_{j}(\mathbf{x}) \mathbf{y}_{j}\right),
$$

so by the equicontinuity of $\left\{\mathbf{T}^{m}: m \in \mathbb{N}\right\}$ the convergence

$$
\mathbf{T}^{s+d m}(\mathbf{x}) \rightarrow \sum_{j=1}^{r} \xi_{j}(\mathbf{x}) \mathbf{y}_{j}
$$

holds. Let us denote by $\Lambda_{j}(\mathbf{x})$ the scalar $\xi_{\alpha^{s-d}(j)}(\mathbf{x})$. It is easy to see that $\mathbf{T}^{d m}(\mathbf{x})$ converges to $\sum_{j=1}^{r} \Lambda_{j}(\mathbf{x}) \mathbf{y}_{j}$ if $m \rightarrow \infty$. Since arbitrary natural number $n$ can be represented as $d m_{n}+s$ where $0 \leq s \leq d-1$,

$$
\begin{aligned}
\mathbf{T}^{n}(\mathbf{x}) & =\mathbf{T}^{s} \mathbf{T}^{d m_{n}}(\mathbf{x}) \\
& =\mathbf{T}^{s}\left(\sum_{j=1}^{r} \Lambda_{j}(\mathbf{x}) \mathbf{y}_{j}+\left(\mathbf{T}^{d m_{n}}(\mathbf{x})-\sum_{j=1}^{r} \Lambda_{j}(\mathbf{x}) \mathbf{y}_{j}\right)\right) \\
& =\sum_{j=1}^{r} \Lambda_{j}(\mathbf{x}) \mathbf{y}_{\alpha^{s}(j)}+\mathbf{R}_{n}(\mathbf{x})=\sum_{j=1}^{r} \Lambda_{j}(\mathbf{x}) \mathbf{y}_{\alpha^{n}(j)}+\mathbf{R}_{n}(\mathbf{x})
\end{aligned}
$$

where $\mathbf{R}_{n}(\mathbf{x})$ tends to $\mathbf{0}$ in the $F$-norm. Now it remains to show that $\Lambda_{j}$ are linear. Let $\mathbf{x}, \mathbf{z} \in \mathbf{X}$ be arbitrary. Because

$$
\begin{aligned}
& \mathbf{0}= \lim _{n \rightarrow \infty} \mathbf{T}^{n}\left(\mathbf{x}+\mathbf{z}-\sum_{j=1}^{r} \Lambda_{j}(\mathbf{x}+\mathbf{z}) \mathbf{y}_{j}\right) \\
&=\lim _{n \rightarrow \infty}\left(\mathbf{T}^{n}\left(\mathbf{x}-\sum_{j=1}^{r} \Lambda_{j}(\mathbf{x}) \mathbf{y}_{j}\right)+\mathbf{T}^{n}\left(\mathbf{z}-\sum_{j=1}^{r} \Lambda_{j}(\mathbf{z}) \mathbf{y}_{j}\right)\right. \\
& \quad+\mathbf{T}^{n}\left(\sum_{j=1}^{r}\left(\Lambda_{j}(\mathbf{x})+\Lambda_{j}(\mathbf{z})-\Lambda_{j}(\mathbf{x}+\mathbf{z})\right) \mathbf{y}_{j}\right),
\end{aligned}
$$

so the third component must converge to $\mathbf{0}$. But this is only possible if it is $\mathbf{0}$. Since $\mathbf{T}_{\Omega}$ is an isomorphism on $\Omega$ and $\mathbf{y}_{j}$ are linearly independent, for every $j$ we have $\Lambda_{j}(\mathbf{x})+\Lambda_{j}(\mathbf{z})-\Lambda_{j}(\mathbf{x}+\mathbf{z})=\mathbf{0}$ and additivity of $\Lambda_{j}$ is proved. By the linearity of $\mathbf{T}$, the homogeneity of $\Lambda_{j}$ is easily seen. The boundedness of $\Lambda_{j}$ is a consequence of the equicontinuity of $\left\{\mathbf{T}^{m}: m \in \mathbb{N}\right\}$. In fact if $\mathbf{x}_{n} \rightarrow \mathbf{0}$ then $\sup _{m \in \mathbb{N}}\left\|\mathbf{T}^{m}\left(\mathbf{x}_{n}\right)\right\|$ tends to 0 . In particular the diameters of $\omega\left(\mathbf{x}_{n}\right)$ tend to 0 and since $\Lambda_{j}\left(\mathbf{x}_{n}\right)$ are coefficients in expansions of some vectors from $\omega\left(\mathbf{x}_{n}\right)$ with respect to the base $\mathbf{y}_{1}, \ldots, \mathbf{y}_{r}$, it follows that $\Lambda_{j}\left(\mathbf{x}_{n}\right) \rightarrow 0$ for every $j$. Positivity of $\Lambda_{j}$ can be easily inferred from the positivity of the coefficients $\xi_{j}(\mathbf{x})$ for positive $\mathbf{x}$. 
(iii) Let $i, k \in\{1, \ldots, r\}$ be arbitrary. We have already proved that

$$
\mathbf{y}_{i}=\mathbf{T}^{n d}\left(\mathbf{y}_{i}\right)=\sum_{j=1}^{r} \Lambda_{j}\left(\mathbf{y}_{i}\right) \mathbf{y}_{j}+\mathbf{R}_{n d}\left(\mathbf{y}_{i}\right)
$$

and the remainder $\mathbf{R}_{n d}\left(\mathbf{y}_{i}\right) \rightarrow \mathbf{0}$, so $\mathbf{y}_{i}=\sum_{j=1}^{r} \Lambda\left(\mathbf{y}_{i}\right) \mathbf{y}_{j}$. Now the property (iii) follows from the independence of $\left\{\mathbf{y}_{1}, \ldots, \mathbf{y}_{r}\right\}$.

(iv) Let $\mathbf{T}^{*}$ be the adjoint operator and $\mathbf{x} \in \mathbf{X}$ be arbitrary. For a fixed $\Lambda_{k}$, $k \in\{1, \ldots, r\}$, we have

$$
\begin{aligned}
\mathbf{T}^{* n} \Lambda_{k}(\mathbf{x}) & =\sum_{j=1}^{r} \Lambda_{j}(\mathbf{x}) \Lambda_{k}\left(\mathbf{y}_{\alpha^{n}(j)}\right)+\Lambda_{k}\left(\mathbf{R}_{n}(\mathbf{x})\right) \\
& =\sum_{j=1}^{r} \Lambda_{j}(\mathbf{x}) \Lambda_{\alpha^{n}\left(\alpha^{-n}(k)\right)}\left(\mathbf{y}_{\alpha^{n}(j)}\right)+\Lambda_{k}\left(\mathbf{R}^{n}(\mathbf{x})\right) \\
& =\Lambda_{\alpha^{-n}(k)}(\mathbf{x})+\Lambda_{k}\left(\mathbf{R}_{n}(\mathbf{x})\right) .
\end{aligned}
$$

This means that $\omega^{*}-\lim _{m \rightarrow \infty} \mathbf{T}^{* m d} \Lambda_{k}=\Lambda_{k}$. Now

$\mathbf{T}^{*} \Lambda_{k}(\mathbf{x})=\lim _{m \rightarrow \infty} \mathbf{T}^{*(m d+1)} \Lambda_{k}(\mathbf{x})=\Lambda_{\alpha^{-1}(k)}(\mathbf{x})+\lim _{m \rightarrow \infty} \Lambda_{k}\left(\mathbf{R}_{m d+1}(\mathbf{x})\right)=\Lambda_{\alpha^{-1}(k)}(\mathbf{x})$,

so (iv) is proved.

(v) Let $\mathbf{x}^{*} \in \mathbf{X}^{*}$ and $\mathbf{x} \in \mathbf{X}$ be arbitrary. Since $\mathbf{T}^{* n}\left(\mathbf{x}^{*}\right)(\mathbf{x})=\mathbf{x}^{*}\left(\mathbf{T}^{n}(\mathbf{x})\right)=$ $\sum_{j=1}^{r} \Lambda_{j}(\mathbf{x}) \mathbf{x}^{*}\left(\mathbf{y}_{\alpha^{n}(j)}\right)+\mathbf{x}^{*}\left(\mathbf{R}_{n}(\mathbf{x})\right)=\sum_{j=1}^{r} \Lambda_{\alpha^{-n}(j)}(\mathbf{x}) \mathbf{x}^{*}\left(\mathbf{y}_{j}\right)+\mathbf{R}_{n}^{*}\left(\mathbf{x}^{*}\right)(\mathbf{x})$ and $\mathbf{R}_{n}(\mathbf{x})$ $\rightarrow \mathbf{0}$ in the $F$-norm, the proof of $(\mathrm{v})$ and the whole Theorem is completed.

Corollary 2.2. Positive and constrictive operators acting in Banach lattices are asymptotically periodic.

Definition 2.4. An operator $\mathbf{T}$ acting on an $F$-space $\mathbf{X}$ is called asymptotically stable if there exist a linear functional $\Lambda \in \mathbf{X}^{*}$ and a vector $\mathbf{y}_{0} \in \mathbf{X}$ such that $\lim _{n \rightarrow \infty} \mathbf{T}^{n}(\mathbf{x})=\Lambda(\mathbf{x}) \mathbf{y}_{0}$ for every $\mathbf{x} \in \mathbf{X}$.

The following result can be easily deduced from our Theorem. In fact, asymptotic stability holds for a constrictive operator if and only if $r=\operatorname{dim}(\Omega)=1$. We notice that the functional $\Lambda$ must be positive for positive $\mathbf{T}$.

Corollary 2.3. Let $\left(\mathbf{X}, \mathbf{X}_{+}\right)$and $\mathbf{T}$ be as in the theorem. If for each $\mathbf{x}_{1}, \mathbf{x}_{2} \in \mathbf{X}_{+}$ with $\mathbf{T}^{n}\left(\mathbf{x}_{i}\right) \nrightarrow \mathbf{0}(i=1,2)$ there exists $\mathbf{z} \in \mathbf{X}_{+}$such that $\mathbf{z} \leq \mathbf{T}^{m}\left(\mathbf{x}_{i}\right)(i=1,2)$ for some natural $m$ and $\mathbf{T}^{n}(\mathbf{z}) \nrightarrow \mathbf{0}$ then $\mathbf{T}$ is asymptotically stable.

3.

Finally we apply our results to the Banach lattice of real valued and twice continuously differentiable functions on $[0,1]$, equipped with the norm $\|g\|_{2}=$ $\max _{0 \leq j \leq 2}\left\|D^{j} g\right\|_{\text {sup }}$ and the positive cone $\mathbf{C}_{+}^{2}([0,1])=\left\{f \in \mathbf{C}^{2}([0]):, D^{j} f \geq 0\right.$ for $j=0,1,2\}$. Here $D^{j}$ denotes the $j$ th differential operator and $\|\cdot\|_{\text {sup }}$ denotes the supremum norm in $\mathbf{C}([0,1])$. For $i \in\{0,1,2, \ldots\}$ the function $e_{i}:[0,1] \rightarrow \mathbb{R}$ is the $i$ th monomial (i.e., $e_{i}(t)=t^{i}$ ). We emphasise that in the sequel the inequality $\preceq$ corresponds to the order introduced by the cone $\mathbf{C}_{+}^{2}([0,1])$ in contrast with $\leq$, which is related to real numbers. By Corollary 2.2 constrictive and positive operators on $\mathbf{C}^{2}([0,1])$ are asymptotically periodic, so we have 
Proposition 3.1. Let $\mathbf{T}$ be a positive and constrictive linear operator on the Banach lattice $\mathbf{C}^{2}([0,1])$. If the functions $e_{0}, e_{1}, e_{2}$ are $\mathbf{T}$-recurrent (i.e., they belong to $\Omega$ ) and $e_{2}$ is an atom in $\Omega$ then:

(3.1) the atoms of $\Omega$ are exactly $e_{0}, e_{1}, e_{2}$;

(3.2) there exists a probability (Radon) measure $\mu$ on $[0,1]$ such that $\lim _{n \rightarrow \infty} \mathbf{T}^{6 n}(f)=f(0) e_{0}+D^{1} f(0) e_{1}+\left(\frac{1}{2} \int D^{2} f d \mu\right) e_{2}$ for every $f \in \mathbf{C}^{2}([0,1])$.

Proof. We begin with the remark that $e_{0}$ and $e_{1}$ are atoms in the whole $\mathbf{C}^{2}([0,1])$ space, so they are atoms in $\Omega$ as well. Let $f$ denote some atom in $\Omega$ that is orthogonal to $e_{0}$ and $e_{1}$. Notice that $f(0)=D^{1} f(0)=0$. We may also assume that $\left\|D^{2} f\right\|_{\text {sup }} \leq 1 \leq D^{2} e_{2} \equiv 2$. Since $D^{1} f(t)=\int_{0}^{t} D^{2} f(w) d w \leq$ $t \leq 2 t=D^{1} e_{2}(t)$ and $f(s)=\int_{0}^{s} D^{1} f(t) d t \leq \int_{0}^{s} 2 t d t=s^{2}=e_{2}(s)$, it follows that $f \preceq e_{2}$. The last relation can occur only if $f=t e_{2}$ for some $t \in[0,1]$ as it is assumed that $e_{2}$ is an atom and (3.1) is proved.

Now, by our Theorem there are linear and positive functionals $\Lambda_{0}, \Lambda_{1}$, $\Lambda_{2}$ from $\mathbf{C}^{2}([0,1])^{*} \cong \mathbb{R}^{2} \times \mathbf{C}([0,1])^{*}$ such that for every $f \in \mathbf{C}^{2}([0,1])$ we have $\mathbf{T}^{6 n} f \rightarrow \Lambda_{0}(f) e_{0}+\Lambda_{1}(f) e_{1}+\Lambda_{2}(f) e_{2}$ in the $\|\cdot\|_{2}$ norm. The Riesz Representation Theorem implies $\Lambda_{i}(f)=\alpha_{i} f(0)+\beta_{i} D^{1} f(0)+\frac{1}{2} \int D^{2} f d \mu_{i}$ for some positive (Radon) measure $\mu_{i}$ on $[0,1](i=0,1,2)$. Since $\Lambda_{i}\left(e_{k}\right)=$ $\delta_{i, k}$ (see (iii)), $\Lambda_{0}(f)=f(0), \Lambda_{1}=D^{1} f(0)$, and $\Lambda_{2}(f)=\frac{1}{2} \int D^{2} f d \mu$ for some probability (Radon) measure $\mu$ on $[0,1]$, so $(3.2)$ is proved.

Remark 3.1. In the above proposition the function $e_{2}$ can be replaced by any function $f$ with the property $D^{2} f(t)>0$ for $t \in[0,1]$.

Remark 3.2. The atomness of $e_{2} \in \Omega$ in the Proposition 2.1 holds when the following condition is imposed on $\mathbf{T}$ : if $f \in \mathbf{C}_{+}^{2}([0,1])$ is nonzero then $\mathbf{0} \notin$ $\omega(f)$ (we say that $\mathbf{T}$ is strongly positive). Indeed, assume that $e_{2}=\sum_{j=1}^{r} \alpha_{j} y_{j}$ where $\left\{y_{1}, \ldots, y_{r}\right\}$ is the atomic base in $\Omega$ and $e_{0}, e_{1} \in \Omega$. Without loss of generality we claim $y_{1}=e_{0}$ and $y_{2}=e_{1}$ so $\alpha_{1}=\alpha_{2}=0$. If $e_{2}$ is not an atom in $\Omega$ then there are at least two different indices $j, k \geq 3$ such that $D^{2} y_{j} \wedge D^{2} y_{k}$ is positive and nonzero $(\wedge$ denotes here the ordinary minimum in $\mathbf{C}([0,1]))$. We define

$$
f(t)=\int_{0}^{t} \int_{0}^{s} D^{2} y_{j} \wedge D^{2} y_{k}(w) d w d s \geq 0 \quad \text { for } t \in[0,1] .
$$

Clearly the functions $y_{j}, y_{k}$ are greater than $f$. Now, since $\mathbf{T}$ is constrictive and strongly positive, the function $\tilde{f}=\lim _{n \rightarrow \infty} \mathbf{T}^{\text {nd }} f \in \Omega_{+}$is nonzero and satisfies $\tilde{f} \preceq y_{k}, \tilde{f} \preceq y_{j}$. It contradicts $y_{j}, y_{k}$ being orthogonal atoms.

Corollary 3.1. Let $\mathbf{T}$ be a strongly positive and constrictive linear operator on $\mathbf{C}^{2}([0,1])$. If $\mathbf{T} e_{j}=e_{j}$ for $j=0,1,2$ then there exists a probability (Radon) measure $\mu$ on $[0,1]$ such that

(3.3) $\mathbf{T}^{n}(f) \rightarrow f(0) e_{0}+D^{1} f(0) e_{1}+\left(\frac{1}{2} \int D^{2} f d \mu\right) e_{2}$ in $\|\cdot\|_{2}$ norm.

Proof. By Proposition 3.1 and Remark 3.2 the functions $e_{0}, e_{1}, e_{2}$ constitute an atomic base in $\Omega$. Since they are T-invariant, $\left.\mathbf{T}\right|_{\Omega}=\left.\mathbf{I d}\right|_{\Omega}$. Now by our Theorem $d=1$, so (3.3) easily follows from (3.2).

Remark 3.3. Our Corollary 3.3 shows that there are only very few finitedimensional and strongly positive projections $\mathbf{P}$ on $\mathbf{C}^{2}([0,1])$ with invariant 
$e_{0}, e_{1}, e_{2}$. It can be easily inferred from previous results that such a projection must be 3-dimensional and have the form $\mathbf{P}(f)=f(0) e_{0}+D^{1} f(0) e_{1}+$ $\frac{1}{2}\left(\int D^{2} f d \mu\right) e_{2}$ for some probability $\mu$ on $[0,1]$.

We end this note with the following criterion of asymptotic stability in $\mathbf{C}^{2}([0,1])$.

Corollary 3.2. Let $\mathbf{T}$ be a constrictive and positive operator on $\mathbf{C}^{2}([0,1])$. Assume that there exists a $\mathbf{T}$-recurrent function $f_{0} \in \mathbf{C}_{+}^{2}([0,1])$ satisfying

(3.4) $\prod_{j=0}^{2} D^{j} f(t)>0$ for all $f \in \omega\left(f_{0}\right)$ and $t \in[0,1]$.

Then the operator $\mathbf{T}$ is asymptotically stable if and only if

(3.5) for every $g \in \mathbf{C}_{+}^{2}([0,1])$ such that $\mathbf{0} \notin \omega(g)$ there are a natural number $m, f \in \omega\left(f_{0}\right)$ and a positive scalar $r$ with $r f \preceq \mathbf{T}^{m} g$.

Moreover in this case $\lim _{n \rightarrow \infty} \mathbf{T}^{n}(f)=\Lambda(f) f_{0}$ for some positive functional $\Lambda$ on $\mathbf{C}^{2}([0,1])$.

Proof. Assume that the operator $\mathbf{T}$ is asymptotically stable. Then the space $\Omega$ is 1 -dimensional, so $\Omega=\left\{t f_{0}: t \in \mathbb{R}\right\}$. For every positive $g$ with $\mathbf{0} \notin$ $\omega(g)$ we have $\mathbf{T}^{n}(g) \rightarrow \Lambda(g) f_{0}$ where $\Lambda(g)>0$. Since the convergence in $\mathbf{C}^{2}([0,1])$ is equivalent to the convergence of derivatives (in the sup norm), $\left\|D^{j} \mathbf{T}^{m}(g)-\Lambda(g) D^{j} f_{0}\right\|_{\text {sup }}<\varepsilon$ if $m$ is big enough $(j=0,1,2)$. Now if $0<\varepsilon<\frac{\Lambda(g)}{2} \inf _{j=0,1,2} \inf _{x \in[0,1]} D^{j} f_{0}(x)$ then $\frac{\Lambda(g)}{2} f_{0} \preceq \mathbf{T}^{m}(g)$, and (3.5) is proved.

Suppose that the operator $\mathbf{T}$ satisfies (3.5). Since it is constrictive, the orbit $\omega\left(f_{0}\right)$ is finite. It follows from (3.4) that every two functions $f_{1}, f_{2} \in \omega\left(f_{0}\right)$ satisfy $\gamma f_{1} \preceq f_{2}$ for some positive scalar $\gamma$. Let $z, y$ be arbitrary atoms in $\Omega$. It remains to show that $z=t y$ for some positive scalar $t$. By (3.5) there are $m_{y}, m_{z} \in \mathbb{N}$ and $r_{y}, r_{z} \in \mathbb{R}_{+}$such that

$$
r_{y} f_{0} \preceq \mathbf{T}^{m_{y}} y \text { and } \quad r_{z} f_{0} \preceq \mathbf{T}^{m_{z}} z \text {. }
$$

In particular, there are $f_{1}, f_{2} \in \omega\left(f_{0}\right)$ such that $r_{y} f_{1} \preceq y$ and $r_{z} f_{2} \preceq z$ and consequently, we have $\gamma r_{z} f_{1} \preceq z$. Since $z$ and $y$ are atoms, $y=t_{y} f_{1}$ and $z=t_{z} f_{1}$ and the proof is complete.

Remark 3.4. Proposition 3.1 and Corollary 3.1 have their versions in $\mathbf{C}^{n}([0,1])$ (with the positive cone $\left\{f \in \mathbf{C}^{n}([0,1]): f \geq 0, D^{1} f \geq 0, \ldots, D^{n} f \geq 0\right\}$ ). They can be easily proved using similar methods.

\section{ACKNOWLEDGMENTS}

The author is grateful to Grażyna Bartoszek and Professor Anzelm Iwanik for their kindness in pointing out several misprints in the first version of this paper.

\section{REFERENCES}

[B] W. Bartoszek, Asymptotic periodicity of the iterates of positive contractions on Banach lattices, Studia Math. 91 (1988), 179-188.

[DS] C. M. Dafermos and M. Slemrod, Asymptotic behaviour of nonlinear contraction semigroups, J. Funct. Anal. 13 (1973), 97-106.

[KN] J. L. Kelly and I. Namioka, Linear topological spaces, Van Nostrand, Princeton, NJ, 1963.

[K] J. Komornik, Asymptotic periodicity of the iterates of weakly constrictive Markov operators, Tôhoku Math. J. 38 (1986), 15-27. 
[LLY] A. Lasota, T. Y. Li, and J. A. Yorke, Asymptotic periodicity of the iterates of Markov operators, Trans. Amer. Math. Soc. 286 (1984), 751-764.

[LZ] W. A. Luxemburg and A. C. Zaanen, Riesz spaces, vol. I, North-Holland, Amsterdam, 1971.

[M] M. Miklavčič, Asymptotic periodicity of the iterates of positivity preserving operators, Trans. Amer. Math. Soc. 307 (1988), 469-479.

[R] S. Rolewicz, Metric linear spaces, PWN-Polish Scientific Publishers, Warsaw, 1984.

[V] Vu Quoc Phong, Asymptotic almost periodicity and compactifying representations of semigroups, Ukrain. Mat. Zh. 38 (1986), 688-696. (Russian)

Department of Mathematics and Applied Mathematics, Potchefstroom University for Christian Higher Education, 2520 Potchefstroom, South Africa

E-mail address: WSKWKB@PUKVM1.PUK.AC.ZA 